\title{
Special Session on Product Environmental Footprint
}

\author{
Mélanie Guiton and Enrico Benetto
}

\begin{abstract}
The Product Environmental Footprint (PEF) Life Cycle Assessment (LCA) method has been adopted by the European Commission in 2013 as part of the Communication "Building the Single Market for Green Products" [1]. Since then a pilot phase involving about 300 companies, industry associations, NGOs and governments has been launched to develop product category rules for more than 20 different product groups. In this session it was discussed the status of play of the work done till now and its future perspective.
\end{abstract}

\section{Introduction}

In 2010 a coalition of big companies from different sectors asked to the at the time European Commissioner for Environment Janez Potočnik to intervene to solve a problem that was undermining their competitiveness in the European market. The problem was the proliferation of environmental labels and certification schemes that was taking place in different European Member States. From the industry viewpoint this situation constrained them in particular to produce different studies and calculations reporting the environmental performance of the same product in case that product was going to be sold in different countries. This situation was created by the fact that different countries (e.g. France, Italy, United Kingdom, Switzerland) were developing product-related environmental information policies based on similar-but-different methodological approaches (being them ISO 14040-44, ISO 14025, WRI GHG protocol, PAS 2050, BP X30, and many more). The same year the European Council put forward the same request to the Commission, that is to develop a harmonised method to calculate the environmental performance of products along their entire supply chain. Based on this double request the Commission developed the Product Environmental

M. Guiton $(\bowtie) \cdot$ E. Benetto

Department ERIN-Environmental Research and Innovation,

Luxembourg Institute of Science and Technology (LIST),

Life Cycle Sustainability and Risk Assessment, 4422 Belvaux, Luxembourg

e-mail: melanie.guiton@list.lu

(C) The Author(s) 2018

E. Benetto et al. (eds.), Designing Sustainable Technologies,

Products and Policies, https://doi.org/10.1007/978-3-319-66981-6_57 
Footprint (PEF) and Organisation Environmental Footprint (OEF) methods. They were adopted by the Commission in April 2013 [2] and published in May 2013 on the Official Journal of the European Union.

The intention was to provide industry, member states and NGOs with a solid science-based LCA calculation tool that would lead to results more reproducible, reliable and verifiable. The achievement of these ambitious objectives, however, requires the development of product category rules (organisation sectoral rules for the implementation of $\mathrm{OEF}$ ) and a number of additional features like clear rules on verification, communication, data quality, etc. In order to develop and test all these new features the Commission decided to launch, in tight collaboration with all stakeholders, a pilot phase (2013-2017).

During the special session on PEF at LCM 2017 conference the work done during the pilot phase has been analysed from different viewpoints and some preliminary insight about possible future application of PEF/OEF has been drawn.

\section{Summary of the Presentations Delivered}

The first presentation was delivered by An De Schryver from the European Commission. Ms. De Schryver presented the main outcomes of the Environmental Footprint (EF) pilot phase. The EF pilot phase has gathered about 300 stakeholders from worldwide, actively developing Product Environmental Footprint Category Rules (PEFCRs) and Organisation Environmental Footprint Sectoral Rules (OEFSRs). These organisations (companies, industry associations, governments, NGOs) have invested important resources to develop a work that they consider key for their future respective interests. On top of the 300 stakeholders "doing" the work, there have been more than 2000 stakeholders who have been monitoring the developments and participating to the several online consultations run during these years. This has been the first case of such a wide exercise run by the European Commission and surely shows the high political and technical relevance of the dossier.

Ms. De Schryver highlighted the main results achieved during the pilot phase. In particular she stressed the following ones:

- Implementation of the materiality principle. It is well known to LCA practitioners that even for the most complicated products there are a limited number of processes that drives the majority of the impacts. The hotspot analysis procedure developed for PEF allows to identify the most relevant impact categories, life cycle stages, processes, and direct elementary flows, i.e. those contributing to at least $80 \%$ of the environmental impacts. The driving processes will be those for which company and/or site specific data will be requested.

- Implementation of the Data Needs Matrix (DNM). Once the most relevant processes are identified, clear instruction on which data shall be collected and 
what kind of secondary datasets can be used are listed in the DNM, based on the level of operational control the company performing the study has.

- Reduction of costs. One of the barriers towards a wider implementation of LCA, especially in Small and Medium Enterprises (SMEs) has been the cost of implementing such studies, due to a number of factors, including the amount of data to be collected/found, the time requested to do calculations, the costs of verification, the cost to access to secondary LCI database. The implementation of PEF will bring down many of the costs of performing a PEF/LCA study, up to $80 \%$, depending on the specific situations. This result will be achieved thank to the availability of common rules (PEFCRs/OEFSRs), high quality secondary LCI datasets available for free to any user around the world, free availability of the models used, the availability of user-friendly software, of e-learning packages developed in several languages, and clear verification rules.

- Harmonisation of secondary data sources. By having clear modelling requirements for secondary dataset tendered, the PEF initiative is steering the harmonisation of existing LCI commercial databases. The Commission will make available more than 8000 secondary LCI datasets coming from 6 different data providers. They will all have the same modelling requirements for the foreground data, the same energy and transport datasets for the background. Moreover, they will all use the same format and nomenclature, therefore making them directly usable in most of the LCA software available on the market.

The second presentation was delivered by Mr Erwin M. Schau from the European Commission Joint Research Centre. He focused his presentation on the concept of classes of performance, based on the example of the PEFCR on olive oil. One of the novelty introduced by the PEF method is the concept of benchmark, corresponding to the PEF profile of the average product sold in Europe. Each PEFCR includes one or more representative products. Each of them is modelled based on the different production techniques and their respective "weight" in terms of consumption based European market. The representative product therefore represents the weighted average for that group and its PEF profile, calculated and reported in the PEFCR, it is the benchmark for that product group. Therefore, by having this reference publicly available, each producer will be able to know if their own product score better or worse than the benchmark. Once the benchmark is available, the next logical step is to consider the possibility of developing classes of environmental performance. During the pilot phase the stakeholders leading the development of PEFCRs were invited to test this concept, in particular trying to develop 5 classes of performance, where the benchmark is always class $\mathrm{C}$. This is an optional requirement. The approach taken to define the classes of performance was to identify the most relevant parameters (in terms of activity data and emissions) and then identify the theoretical minimum and maximum values possible for such parameters. Once these were identified the calculations were run to identify the range of performance that could be achieved with different combinations of the various parameters. Mr. Schau highlighted the pros and cons of this approach, clearly stating that the ideal solution would be to base the 
definition of the classes of performance on a statistically representative sample of PEF profiles calculated for real products.

The third presentation was delivered by Mr. Quentin De Hults, speaking on behalf of PlasticsEurope. PlasticsEurope has been committed in LCA for decades by managing a dataset program for plastics, and by contributing to LCA developments e.g. through the UNEP SETAC Life Cycle Initiative. PlasticsEurope also believes in LCA to be completed by other tools like risk assessment. With this constructive state of mind, PlasticsEurope is participating to the European Product Environmental Footprint, officially in 3 pilots and in many other working groups and workshops. According to Mr. De Hults the EF pilot phase contributed to improve the best practice in the LCA domain thanks to the transparency of the approach and the high quality of the technical discussions that take place at the Technical Advisory Board. He also mentioned a number of issues to be further improved, referring e.g. to the improvement of the toxicity impact categories, with the development of more reliable characterisation factors. He mentioned that the approach taken in PEF should be complemented with other approaches like Proscale (a specific presentation of Proscale took place during the same conference). Another area of improvement referred to the development of secondary datasets when there are only few companies running certain processes, taking into account the associated industrial confidentiality issues to be addressed. According to PlasticsEurope, there is room for the use of PEF into existing or new product policies provided that it remains a voluntary approach.

The fourth presentation was delivered by Mr. Pau Huguet Ferran from Ecomatters. Mr. Ferran spoke on behalf also of PRé Consultants. The two companies supported the European Commission during the EF pilot phase by reviewing a number of PEF studies carried out by companies on real products to test the feasibility of implementation of the draft PEFCR developed. The shortcomings identified when reviewing these studies have been very important to improve the quality of the PEFCRs and to correct some flaws in the methodological requirements developed during the pilot phase. Some of the most relevant problems highlighted by the reviewers were that (i) the PEFCRs were not strict enough in terms of modelling requirements, (ii) the DNM was not systematically implemented, (iii) the Circular Footprint Formula, that is the single formula to calculate credits and burden related to the end of use of a product, was too complicated and wrongly implemented by some companies, (iv) the way how the representative product has been modelled by the stakeholders leading the PEFCR development is not necessarily supported the companies implementing it.

Based on the outcome of these reviews a number of changes have been implemented in the Guidance documents supporting the pilot phase.

The fifth and last presentation was delivered by Ms. Mihaela Thuring from VITO, institute actively involved in two pilots and some supporting contracts. According to Ms. Thuring environmental information on organisations and products are important tools that companies can use for achieving environmental goals towards a sustainable society. Both ISO 14001 (Environmental management systems) and PEF are aimed at providing such information to market players. 
The PEF method explores a new way of communicating the environmental impacts of products through the application of the PEF formula(s), as part of the efforts towards a Circular Economy. This new way expresses no longer the actual potential environmental impact, but includes also the potential benefits of the recycling/ recoverability process, as well as the energy recovery potential of the materials. While understandable for professionals in the field, this new approach poses multiple questions in determining how this complex message gets across to consumers and how it will contribute to fulfilling the original goal of streamlining the communication of environmental information. The wide participation in the PEF/OEF pilot of actors across most industries and beyond European borders showed that the relevance of LCA methods in taking decisions and communicating on environmental related aspects is more actual than ever, as well as the interest in the unifying approach which the PEF/OEF method is bringing.

VITO stated that the identification of information flows between PEF and ISO 14001 determines how an organisation can integrate both the PEF and ISO 14001 in a coherent and efficient manner.

\section{Conclusions}

The discussion during this special session highlighted the great amount of work done by the European Commission and all stakeholders in the context of the Environmental Footprint pilot phase. It is widely acknowledged that major improvements from the methodological point of view have been achieved, leading to a new generation of LCA/PEF studies that should deliver results that are more reliable, more reproducible and more verifiable. The pilot phase highlighted issues to be solved in the area of characterisation factors in existing impact assessment methods, the need to develop more and better defined impact categories (e.g. in the area of biotic and abiotic resources). There are important quality issues found in all commercial databases that would require paying more attention and, in particular, to the review process of these databases. There is work to be done to better identify the benchmarks, and to develop classes of performance. There is an important "educational" work to be done within practitioners, policy makers, and NGOs when it comes to the development and use of weighting factors. However, notwithstanding all these possible improvements, the LCA community is aware that their credibility depends highly on the success of PEF. There are market needs to be addressed, there are policies that could highly benefit from a consistent use of LCA principle. There are problems related to misleading green claims and the way we implement green public procurement that could be addressed and maybe solved by embedding PEF into a number of existing or new policies. After the pilot phase the European Commission will engage with all interested stakeholders in an open conversation about the future role of PEF/OEF and LCA into European policies. 
Acknowledgements The session on Product Environmental Footprint has been chaired by Mr. Michele Galatola, Product Team Leader at European Commission-DG ENV, and Mr. Alberto Frausin, CEO at Carlsberg Italy. The authors would like to greatly acknowledge Mr. Michele Galatola for having provided the complete content of this paper. As agreed between the declared authors of the paper and Mr. Galatola, he remains the corresponding author Michele.GALATOLA@ec.europa.eu.

\section{References}

1. European Commission, Communication from the Commission to the European Parliament and the Council: Building the Single Market for Green Products-Facilitating better information on the environmental performance of products and organisations, April 2013, COM/2013/0196 final.

2. European Commission, Recommendation of 9 April 2013 on the use of common methods to measure and communicate the life cycle environmental performance of products and organisations, 2013/179/EU.

Open Access This chapter is licensed under the terms of the Creative Commons Attribution 4.0 International License (http://creativecommons.org/licenses/by/4.0/), which permits use, sharing, adaptation, distribution and reproduction in any medium or format, as long as you give appropriate credit to the original author(s) and the source, provide a link to the Creative Commons license and indicate if changes were made.

The images or other third party material in this chapter are included in the chapter's Creative Commons license, unless indicated otherwise in a credit line to the material. If material is not included in the chapter's Creative Commons license and your intended use is not permitted by statutory regulation or exceeds the permitted use, you will need to obtain permission directly from the copyright holder. 